\title{
Progressive Changes in Walking Kinematics and Kinetics After Anterior Cruciate Ligament Injury and Reconstruction: A Review and Meta-Analysis
}

\author{
Lindsay V. Slater, PhD*; Joseph M. Hart, PhD, ATC, FNATA, FACSM*; \\ Adam R. Kelly, MS†; Christopher M. Kuenze, PhD, ATC†
}

*Department of Kinesiology, The University of Virginia, Charlottesville; †Department of Kinesiology, Michigan State University, East Lansing

Context: Anterior cruciate ligament (ACL) injury and $A C L$ reconstruction (ACLR) result in persistent alterations in lower extremity movement patterns. The progression of lower extremity biomechanics from the time of injury has not been described.

Objective: To compare the 3-dimensional (3D) lower extremity kinematics and kinetics of walking among individuals with ACL deficiency (ACLD), individuals with ACLR, and healthy control participants from 3 to 64 months after ACLR.

Data Sources: We searched PubMed and Web of Science from 1970 through 2013.

Study Selection and Data Extraction: We selected only articles that provided peak kinematic and kinetic values during walking in individuals with ACLD or ACLR and comparison with a healthy control group or the contralateral uninjured limb.

Data Synthesis: A total of 27 of 511 identified studies were included. Weighted means, pooled standard deviations, and 95\% confidence intervals were calculated for the healthy control, ACLD, and ACLR groups at each reported time since surgery. The magnitude of between-groups (ACLR versus ACLD, control, or contralateral limb) differences at each time point was evaluated using Cohen d effect sizes and associated 95\% confidence intervals. Peak knee-flexion angle (Cohen $\mathrm{d}=-0.41$ ) and external knee-extensor moment (Cohen $d=-0.68$ ) were smaller in the ACLD than in the healthy control group. Peak knee-flexion angle (Cohen $d$ range $=-0.78$ to -1.23 ) and external knee-extensor moment (Cohen $\mathrm{d}$ range $=-1.39$ to -2.16) were smaller in the ACLR group from 10 to 40 months after ACLR. Reductions in external knee-adduction moment (Cohen $d$ range $=-0.50$ to -1.23 ) were present from 9 to 42 months after ACLR.

Conclusions: Reductions in peak knee-flexion angle, external knee-flexion moment, and external knee-adduction moment were present in the ACLD and ACLR groups. This movement profile during the loading phase of gait has been linked to knee-cartilage degeneration and may contribute to the development of osteoarthritis after ACLR.

Key Words: gait biomechanics, knee osteoarthritis, anterior cruciate ligament deficiency, movement dysfunction

\section{Key Points}

- Alterations in frontal- and sagittal-plane walking kinematics and kinetics persisted for 12 to 36 months after anterior cruciate ligament reconstruction.

- These gait patterns did not appear to normalize over time despite the individual's return to physical activity after anterior cruciate ligament reconstruction, indicating that those with dysfunctional movement patterns are not adequately identified using current approaches to rehabilitation and assessment before return to activity.

A fter anterior cruciate ligament (ACL) injury, patients and clinicians must determine the optimal treatment approach to ensure joint stability and promote a return to normal lower extremity function. Anterior cruciate ligament reconstruction (ACLR) is a common treatment after initial injury to restore joint stability and return patients to preinjury levels of physical activity. An estimated 250000 ACL injuries and 130000 ACLR procedures occur annually in the United States. ${ }^{1}$ Many patients delay or forego surgery, opting for a course of rehabilitation with the hope of returning to activity with ACL deficiency (ACLD). ${ }^{2}$ Regardless of the treatment plan, many physically active individuals with ACLD or ACLR experience persistent reductions in knee function, ${ }^{3}$ physical performance, ${ }^{4}$ and physical activity. ${ }^{2,5}$
Many individuals experience persistent deficits in lower extremity neuromuscular function ${ }^{5-7}$ and alterations in lower extremity movement patterns after ACLR, which may substantially affect global lower extremity performance during activities of daily living and recreational physical activity. ${ }^{8-11}$ More troubling, this cohort of patients has a 15 times increased risk of subsequent ACL injury ${ }^{12}$ and a 3 to 4 times increased risk of posttraumatic knee-joint osteoarthritis compared with uninjured individuals. ${ }^{13} \mathrm{Sev}-$ eral authors have hypothesized that cartilage-loading patterns or surrogate measures of knee-joint health, such as substantial alterations in 3-dimensional (3D) lower extremity joint angles or peak joint moments, may provide key information about the source of poor short- and longterm outcomes after ACLR. ${ }^{14,15}$ Assessing and comparing 
characteristics of lower extremity movement in those with ACLR may provide a more quantitative approach to identifying patients at elevated risk for reinjury and poor long-term joint health. ${ }^{14,16}$

Researchers ${ }^{14,16,17}$ have hypothesized about which gait characteristics are important for identifying patients at increased risk of injury or developing knee-joint osteoarthritis after ACLR. Reductions in peak knee-flexion angle and external knee-flexion moment during the loading phase of walking gait have been consistently reported at 6 to 60 months after ACLR. ${ }^{9,18,19}$ However, it is unclear if these alterations coincide with increases or decreases in peak external knee-adduction moment or peak internal-rotation angle, both of which have been directly and consistently related to patterns of knee-joint cartilage loading and degeneration, over this period. ${ }^{14,16,20-22}$ A clearer understanding of the progression of 3D lower-extremity biomechanics from the time of injury through long-term followup may help clinicians and researchers better target evaluation and intervention to improve short- and longterm outcomes after ACLR. Therefore, the purpose of our review was to present the best current evidence about 3D walking kinematics and kinetics of the hip and knee joints in individuals with ACLD, individuals with a history of ACLR, and those serving as healthy control participants. In addition, we organized and consolidated the available evidence based on the time since ACLR to compare variables at both short- and long-term follow-up points after ACLR.

\section{METHODS}

\section{Study Selection and Data Extraction}

We performed a Web-based search using PubMed and Web of Science from 1970 through 2013 with the following search queries to find potential articles: $(A C L$ reconstruction OR anterior cruciate ligament reconstruction OR $A C L$ injury OR $A C L$ deficiency OR $A C L$ insufficiency) AND (gait OR walking). The titles of all returned records were reviewed for relevance, and those deemed potentially relevant were retained for further review. In addition, the reference sections of all potentially relevant articles were reviewed to identify other potential articles. Two authors (L.V.S., A.R.K.) evaluated the articles for inclusion, and 1 author (C.M.K.) arbitrated in case of disagreement. Included articles were limited to those presenting means and standard deviations (SDs) for the peak kinematic and kinetic variables of interest during walking, as well as time since injury or surgery in those with ACLD or a history of ACLR. In addition, articles had to include a comparison with a healthy control group or the contralateral uninjured limb. If means and SDs were not available, we contacted the corresponding author for the given article to procure the relevant data. If the data were not available or the author did not respond, the article was excluded from this review. We evaluated the quality of the included studies using the Physiotherapy Evidence Database (PEDro) scale and the Downs and Black $^{23}$ checklist score. The PEDro scale is widely used and appropriate for clinical trials, whereas the Downs and Black $^{23}$ checklist score was designed for use in noninterventional research. Given that both are widely used and have consistently been shown to be effective evaluation tools for methodologic quality, all included articles were scored using both. ${ }^{24,25}$

\section{Data Analysis}

Studies reporting the peak joint angles or moments for the knee and hip joints during the stance phase of walking gait were retained for analysis. ${ }^{9,17,19,26-48}$ Given the variation in units of measure and presentation of data, only studies reporting external kinetics during early stance and normalized to body weight and height $(\mathrm{Nm} / \% \mathrm{BW} \times \mathrm{Ht})$ were included. Data were organized by group (ACLR, ACLD, or healthy control) and limb (involved or contralateral). For the ACLR involved limb, we subsequently grouped data based on the average time since surgery as presented in the article. We calculated weighted averages based on the sample size of each study and used these averages to calculate a point estimate for each group (healthy control, ACLR contralateral limb, ACLD, or ACLD contralateral limb) and each time point after ACLR. Applying the sample size of each study, we calculated the weighted variance for each time point or comparison group and used this value to calculate the SD estimate and 95\% confidence interval (CI) for each mean estimate. Whereas gait speed was a potential confounder, we did not control for this variable, as individual participant data were not available for normalization of kinematic and kinetic variables within each included study.

The magnitude of difference between the ACLR involved limb at each time point since surgery and both the ACLR contralateral limb and the healthy control group was estimated using Cohen $\mathrm{d}$ effect sizes via the following formula:

$$
\text { Cohen } \mathrm{d}=\frac{\mathrm{Mean}_{\text {injured }}-\mathrm{Mean}_{\text {control }}}{\mathrm{SD}_{\text {pooled }}} \text {. }
$$

Control represents either the contralateral limb or the healthy control group. Cohen d effect sizes were interpreted as weak $(<0.21)$, small $(0.21-0.39)$, medium $(0.4-0.7)$, or large $(>0.7)$. In addition, the $95 \%$ CI was calculated for each effect size to establish between-groups or betweenlimbs differences. In all cases, if the $95 \%$ CI did not cross zero, the effect size was considered different. We analyzed the data using Excel (version 14.7.3; Microsoft Corp, Redmond, WA).

\section{RESULTS}

\section{Included Studies and Methodologic Quality Assessment}

Our initial searches identified 511 studies, of which 47 were eligible for inclusion (Figure 1). We excluded 20 studies because they included nonwalking tasks, did not include means and SDs for group comparisons, or used different normalization methods for kinetic data. The 27 included studies had an average PEDro scale score of 4.74 and an average modified Downs and Black ${ }^{23}$ checklist score of 18.26 (Table 1). The average walking speed for individuals with ACL injuries and ACLR was $1.38 \pm$ $0.25 \mathrm{~m} / \mathrm{s}($ range $=0.83-2.00 \mathrm{~m} / \mathrm{s})$. 


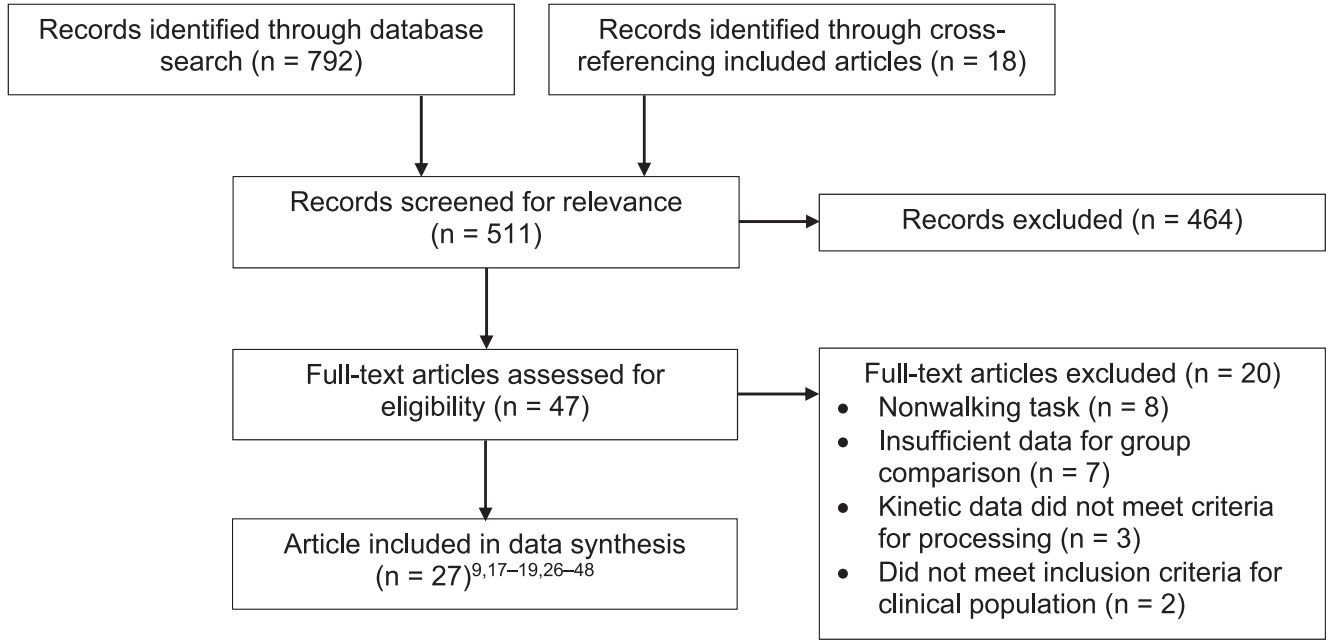

Figure 1. Preferred Reporting Items for Systematic Reviews and Meta-Analyses (PRISMA) flow chart depicting the search strategy and process of study retention, which was completed July 1, 2015.

\section{Outcomes of ACLD}

The ACLD group displayed a large reduction in peak external knee-flexion moment (Cohen $\mathrm{d}=-0.97 ; 95 \% \mathrm{CI}=$ $-1.39,-0.54$; Figure 2 ) and hip-adduction angle (Cohen $\mathrm{d}=$ $-0.82 ; 95 \% \mathrm{CI}=-1.44,-0.20)$, along with moderate reductions in peak knee-flexion angle (Cohen $\mathrm{d}=-0.60$; $95 \% \mathrm{CI}=-0.85,-0.36$; Figure 3 ) and external kneeadduction moment (Cohen $\mathrm{d}=-0.69 ; 95 \% \mathrm{CI}=-1.23$, -0.15 ; Figure 4) compared with the healthy control group (Tables 2 and 3).

\section{Outcomes of ACLR}

Kinematics. Peak knee-flexion angle was smaller in the ACLR group than in the healthy control group and the contralateral limb at all time points after reconstruction except at 24 months (Figure 3). Peak hip-flexion angle was greater in the ACLR group than in the healthy control group and the contralateral limb during the 9- to 11-month time frame (Figure 3).

Peak knee-adduction angle was greater in the ACLR group than in the healthy control group and the contralateral limb at 11 and 20 months after ACLR but was smaller than in the healthy control group and the contralateral limb at 48 and 64 months after ACLR (Figure 4). Peak hipadduction angle was also greater in the ACLR group at 64 months after surgery than in the healthy control group (Cohen $\mathrm{d}=0.64 ; 95 \% \mathrm{CI}=0.02,1.26)$ and the contralateral limb (Cohen $d=1.68 ; 95 \% \mathrm{CI}=0.91,2.46$; Table 3). Peak knee internal-rotation angle was smaller in the ACLR group than in the healthy control group and the contralateral limb at 14, 17, and 48 months after ACLR (Figure 5).

Kinetics. Peak external knee-flexion moment was smaller in the ACLR group than in the healthy control group at 10, 11, and 26 months after ACLR and the contralateral limb at 11 and 26 months (Figure 2). Peak external knee-extension moment in the ACLR group was also smaller than in the healthy control group and the contralateral limb at 9, 11, and 26 months after ACLR (Figure 2). Peak external hip-extension moment (Cohen $\mathrm{d}=$ $-0.91 ; 95 \% \mathrm{CI}=-1.62,-0.21)$ and peak external hipflexion moment (Cohen $\mathrm{d}=-0.85 ; 95 \% \mathrm{CI}=-1.56,-0.15$ ) were smaller in the ACLR than in the healthy control group at 11 months after reconstruction (Table 2).

Peak external knee-adduction moment was smaller in the ACLR group than in the healthy control group at 9, 26, and 34 months after ACLR and the contralateral limb at 34 months after reconstruction (Figure 4). Peak external kneeexternal rotation moment was also smaller in the ACLR group than in the healthy control group at 26 (Cohen $d=$ $-0.64 ; 95 \% \mathrm{CI}=-1.06,-0.21)$ and 34 (Cohen $\mathrm{d}=-1.06$; $95 \% \mathrm{CI}=-1.59,-0.54)$ months after ACLR and in the contralateral limb at 34 (Cohen $\mathrm{d}=-0.50 ; 95 \% \mathrm{CI}=-0.97$, -0.03; Table 2) months after ACLR.

\section{DISCUSSION}

The biomechanical effects of ACL injury and ACLR are commonly studied in isolation; however, very few researchers ${ }^{32,46}$ have followed individuals through the full scope of treatment and rehabilitation. To our knowledge, our review is the first attempt to establish a timeline for the progression of lower extremity kinematics and kinetics from the time of initial injury through surgical reconstruction, rehabilitation, and return to physical activity. Based on our findings, it appears that, compared with healthy individuals, those with ACLD experience substantial reductions in peak knee-flexion angle, peak knee-adduction angle, peak external knee-flexion moment, and peak external knee-adduction moment. These adaptations have been linked to muscle weakness, ${ }^{33}$ specifically of the quadriceps in the sagittal plane and the lateral hip musculature in the frontal plane, ${ }^{49,50}$ which is consistent with common clinical findings after ACL injury. ${ }^{51,52}$ Interestingly, in most studies included in this review, these adaptations persisted through the first year after ACLR despite the surgical restoration of knee-joint stability. In addition, peak hip-flexion angle and peak external kneeextension moment differed from those values in healthy individuals. Despite the improvements in objective and patient-reported measures of function that have been consistently reported ${ }^{53}$ over this 12 -month period, frontaland sagittal-plane patterns of ambulation remained altered in ways that may indicate underlying neuromuscular or 
Table 1. Description of Included Studies and Assessment of Methodologic Quality Extended on Next Page



Abbreviations: ACLD, anterior cruciate ligament deficient; ACLR, anterior cruciate ligament reconstructed; NA, not applicable; PEDro, Physiotherapy Evidence Database.

a Score range $=0-10$.

b Score range $=0-32$. 


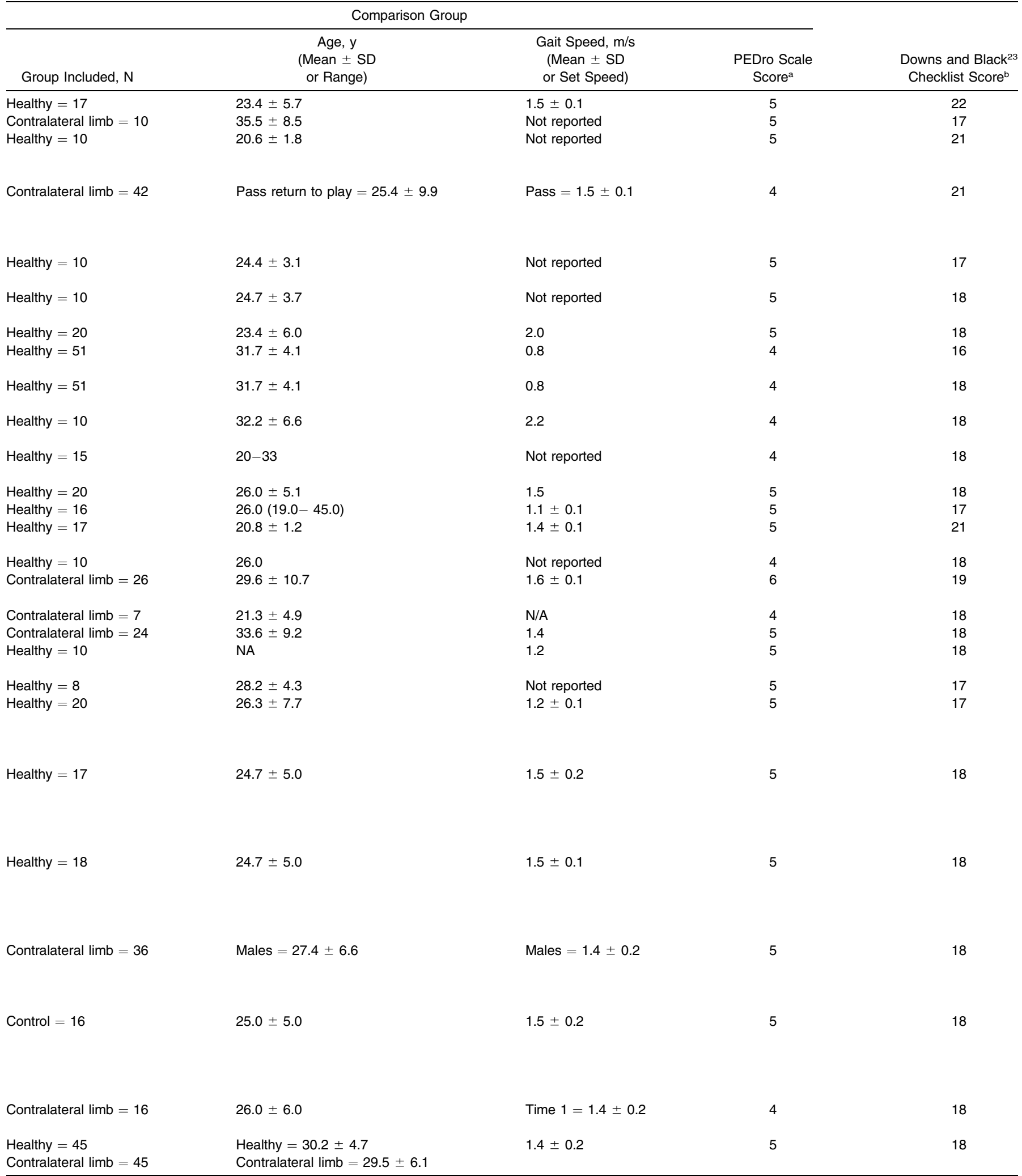

proprioceptive deficits that were not addressed during the rehabilitation process.

It is unlikely that an individual will tear the ACL graft or contralateral ACL while walking. However, this pattern of gait alterations that persists beyond the average time of return to physical activity may indicate high-risk biome- chanics that worsen due to increased task complexity during higher-level tasks, such as jumping and cutting, that are commonly associated with physical activity. The average individual who was previously physically active returns to physical activity about 9 months after ACLR, ${ }^{2}$ and nearly $67 \%$ return to preinjury levels of activity by 2 

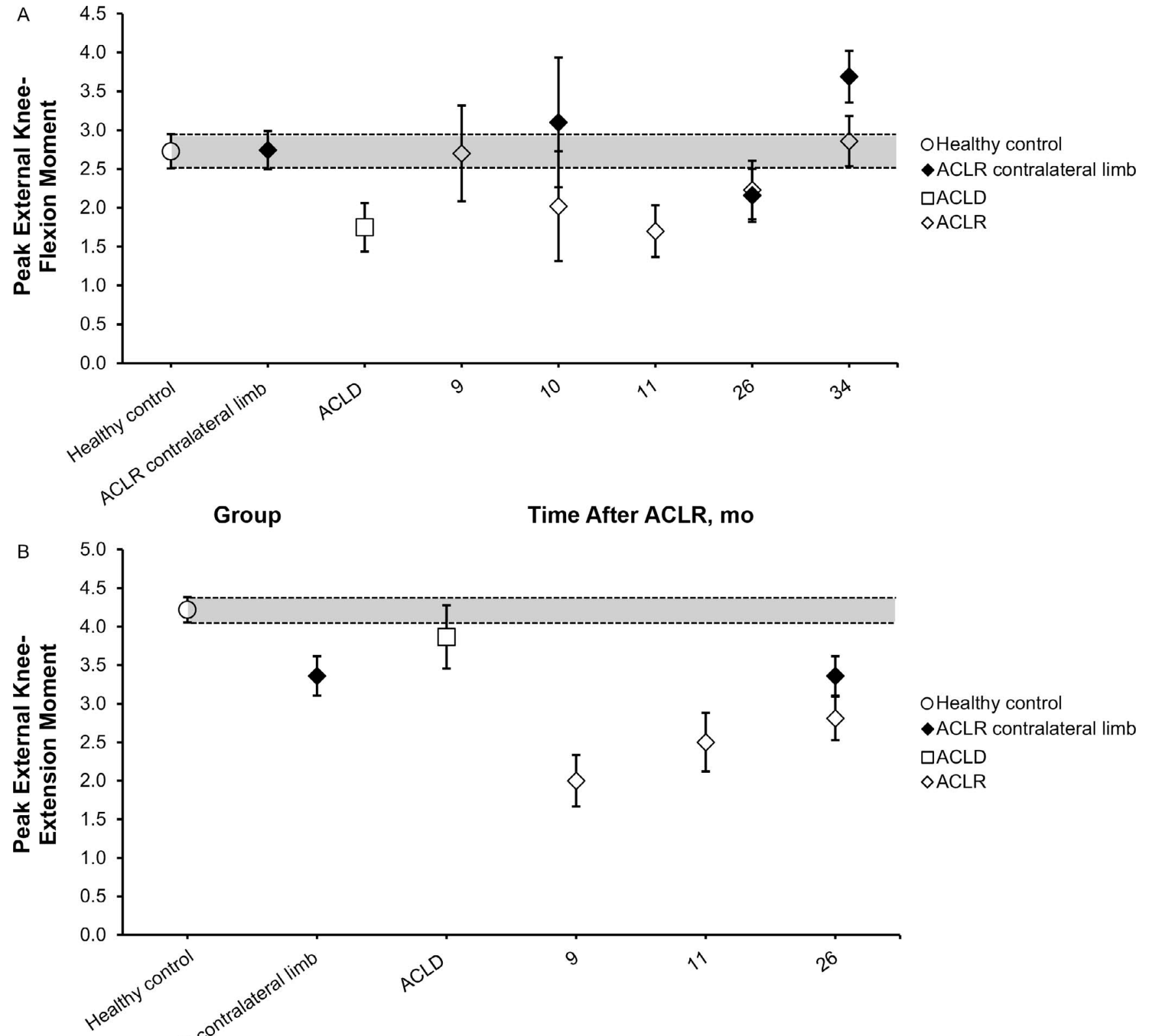

Group

Time After ACLR, mo

Figure 2. A, Peak external knee-flexion moment and, B, knee-extension moment point estimates with $95 \%$ confidence intervals for the healthy control group, anterior cruciate ligament reconstruction (ACLR) contralateral limb group, anterior cruciate ligament deficiency (ACLD) group, and each reported time point after ACLR during stance phase of walking gait. All moments are normalized to percentage of body weight and height. The $95 \%$ confidence interval for the healthy control group is represented using a shaded bar for comparison to all groups and time points.

years after surgery. ${ }^{54,55}$ This trend toward improved physical activity has been reported by multiple researchers; however, many measures of muscle function, ${ }^{6}$ proprioception, ${ }^{56}$ and functional performance ${ }^{57,58}$ do not appear to follow the same trajectory over this period. Based on our findings, many of the gait adaptations in those with ACLD and in the short-term follow-up period after ACLR persist through the midterm ( $<36$ months). Conventional clinical wisdom has been that, as patients reintegrate into physical activity and in some cases competitive sport, indicators of global lower extremity function and coordination, such as gait, should continue to improve. Yet this does not appear to be the case. Reductions in peak knee-flexion angle, peak external knee-flexion moment, peak external knee-extension moment, and peak external knee-adduction moment persisted from short-term follow-up through this midterm period. As the time since surgery increased, the frontal- and sagittal-plane alterations became problematic, not only as potential indicators of underlying traumatic injury risk but also as mechanisms through which knee-joint cartilage degeneration may be accelerated. ${ }^{41,59-61}$ This hypothesis is strengthened by the increased peak knee-external rotation angle and reduced external knee-external rotation moment that were evident at 17 and 34 months, respectively. Alterations in transverse-plane motion have been directly linked to increased cartilage loading and thinning, which 


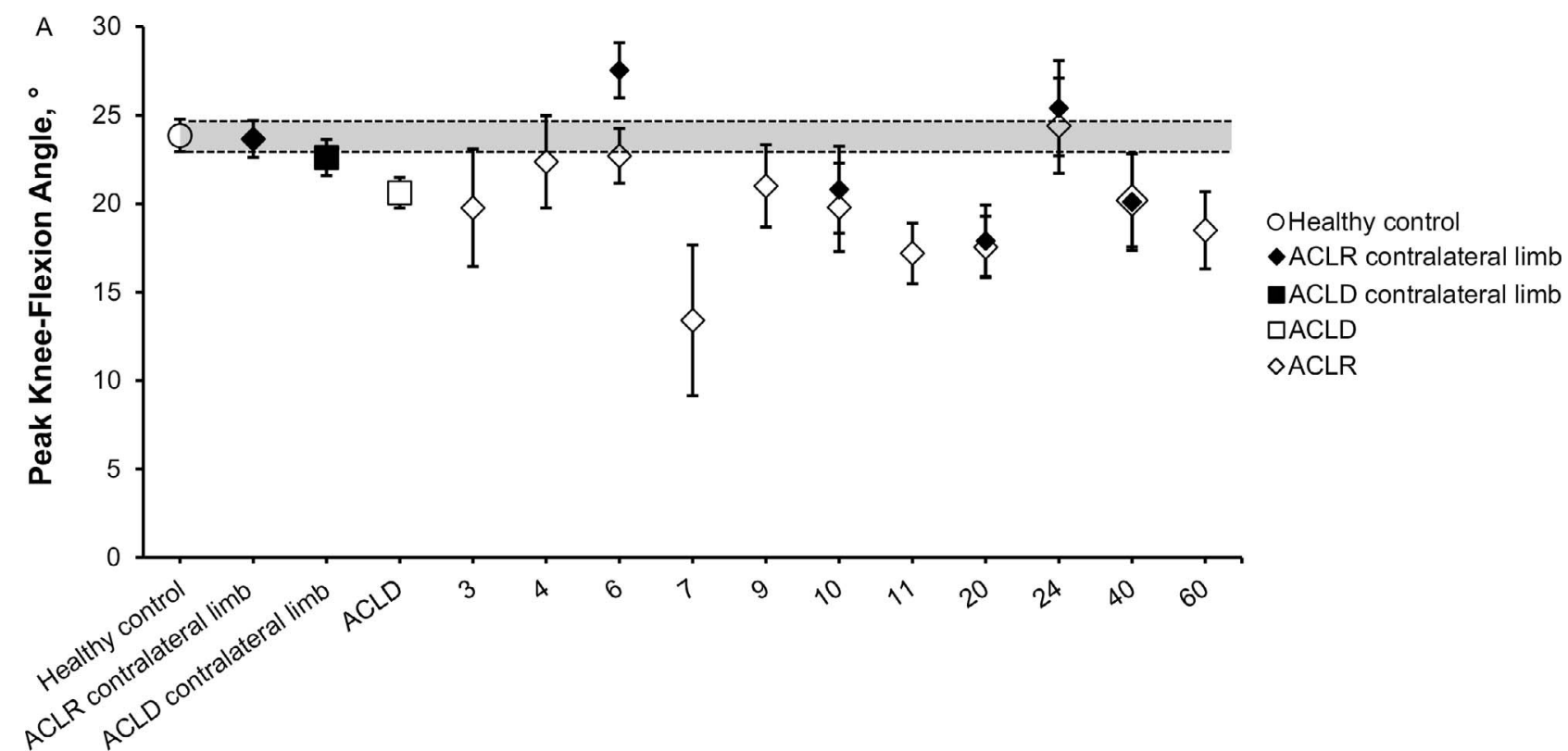

Group Time After ACLR, mo

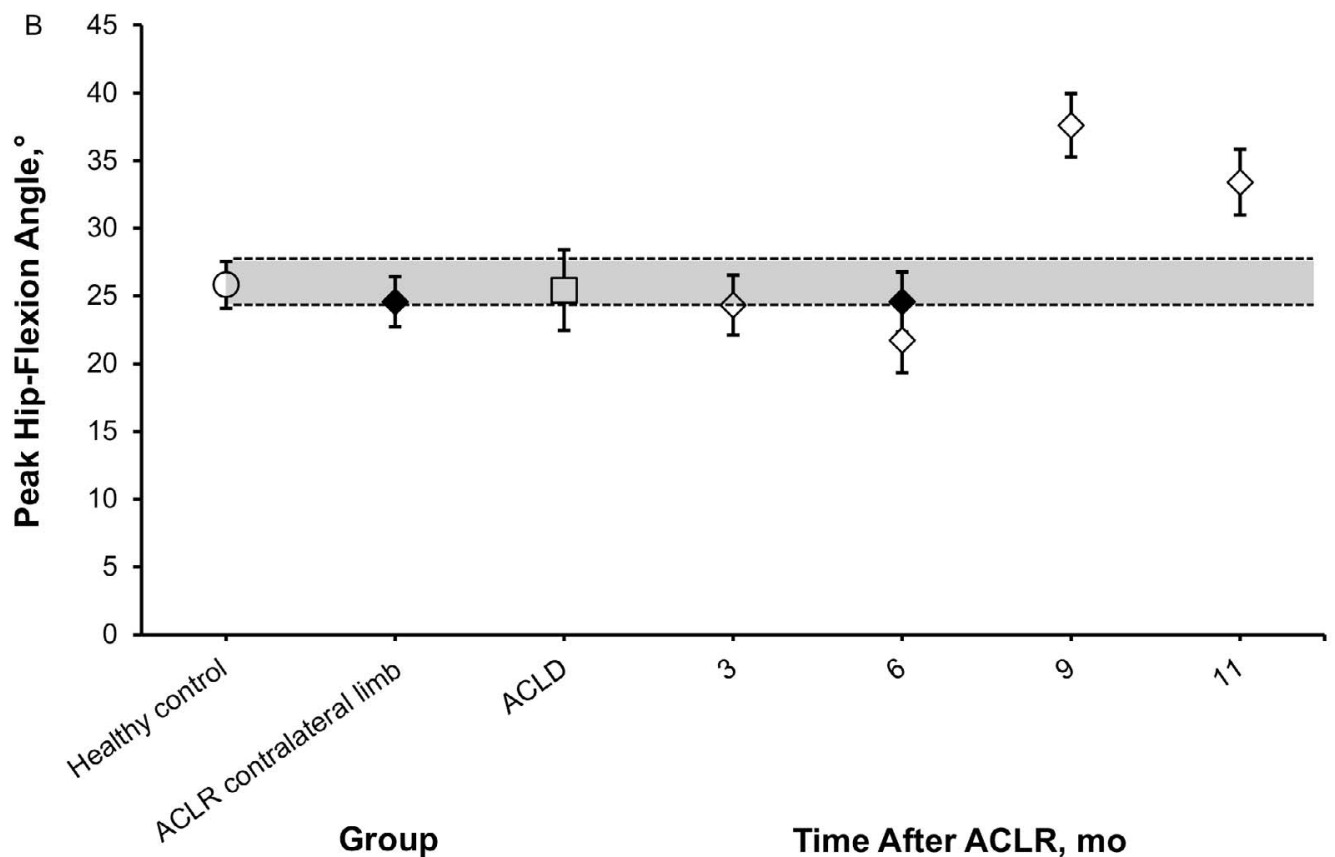

Figure 3. A, Peak knee-flexion angle point estimates with $95 \%$ confidence intervals for the healthy control group, anterior cruciate ligament reconstruction (ACLR) contralateral limb group, anterior cruciate ligament deficient (ACLD) contralateral limb group, ACLD group, and each reported time point after ACLR during the stance phase of walking gait. B, Peak hip-flexion angle point estimates with $95 \%$ confidence intervals for the healthy control group, ACLR contralateral limb group, ACLD group, and each reported time point after ACLR during the stance phase of walking gait. The $95 \%$ confidence interval for the healthy control group is represented using a shaded bar for comparison to all groups and time points.

are potential signs of degeneration over time. ${ }^{16,27,62}$ Continued triplanar alterations in walking gait for a 3-year period after ACLR, which can account for as many as 4 million steps for the average American, ${ }^{63}$ may have a substantial effect on joint health at long-term follow-up.

Data on long-term lower extremity gait biomechanics are limited in the population with ACLR ${ }^{64}$ In our review, the authors of only 2 investigations provided data on patients with a follow-up greater than 36 months after ACLR. ${ }^{27,47}$ Based on these limited findings, those with ACLR may continue to exhibit substantial reductions in peak knee- flexion angle and peak knee-adduction angle as late as 64 months after ACLR; however, insufficient data are available to describe any kinetic differences from healthy individuals. The best currently available evidence continues to point toward substantial and persistent multiplanar alterations in knee-joint kinematics. Given limitations in obtaining prospective data for gait patterns and the progression of knee-joint health, it may be difficult to directly relate these findings, but reductions in peak kneeflexion angle and peak knee-adduction angle have been common findings among individuals with knee-joint 

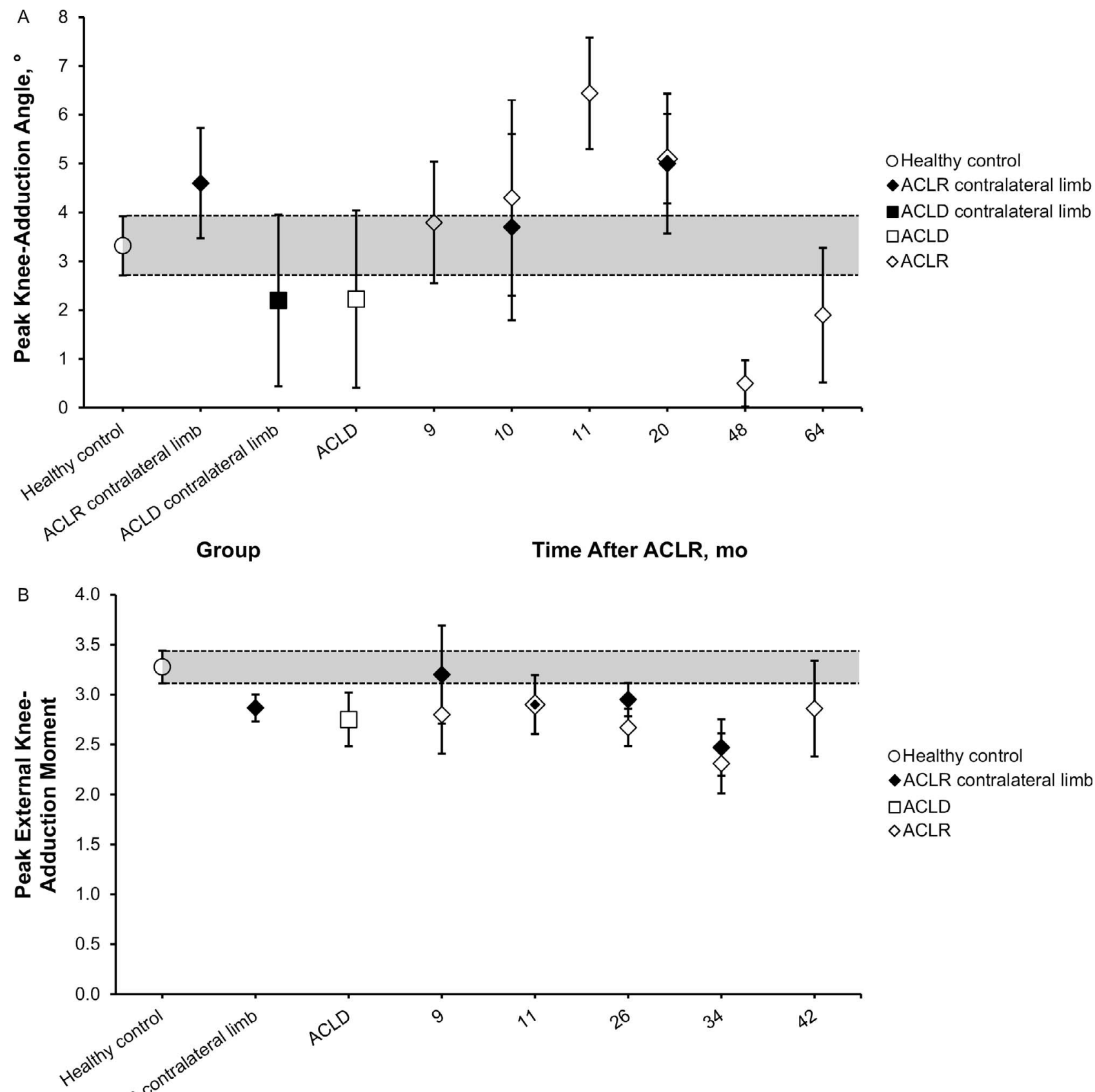

Group

Time After ACLR, mo

Figure 4. A, Peak knee-adduction angle point estimates with $95 \%$ confidence intervals for the healthy control group, anterior cruciate ligament reconstruction (ACLR) contralateral limb group, anterior cruciate ligament deficient (ACLD) contralateral limb group, ACLD group, and each reported time point after ACLR during the stance phase of walking gait. B, Peak external knee-adduction moment point estimates with $95 \%$ confidence intervals for the healthy control group, ACLR contralateral limb group, ACLD group, and each reported time point after ACLR during the stance phase of walking gait. All moments are normalized to percentage of body weight and height. The $95 \%$ confidence interval for the healthy control group is represented using a shaded bar for comparison to all groups and time points.

osteoarthritis in several reviews. ${ }^{61,65}$ Despite increased knee adduction, those with ACLD or ACLR demonstrated decreased external knee-adduction moments, suggesting that they may have walked more slowly to mitigate loading at the knee joint or adopted movement strategies resulting in reduced ground reaction forces in the involved limb compared with the healthy individuals. We described these potential explanations for our findings, but a prospective, systematic investigation into the interaction of knee-joint biomechanics, walking speed, and vertical ground reaction forces after ACLR may provide key insights into the source and expression of altered gait patterns in this population. Increased knee-adduction moment has been hypothesized to be a critical factor leading to knee-joint osteoarthritis, ${ }^{61,66}$ but our results suggest that knee-adduction moment decreases after ACL injury and subsequent reconstruction (Figure 4). We included a timeline up to 42 months after reconstruction, and more research is needed on long-term alterations in peak knee-adduction moment after reconstruction. 
Table 2. Peak External Sagittal-, Frontal-, and Transverse-Plane Knee and Hip Kinetics After Anterior Cruciate Ligament Injury and Subsequent Anterior Cruciate Ligament Reconstruction

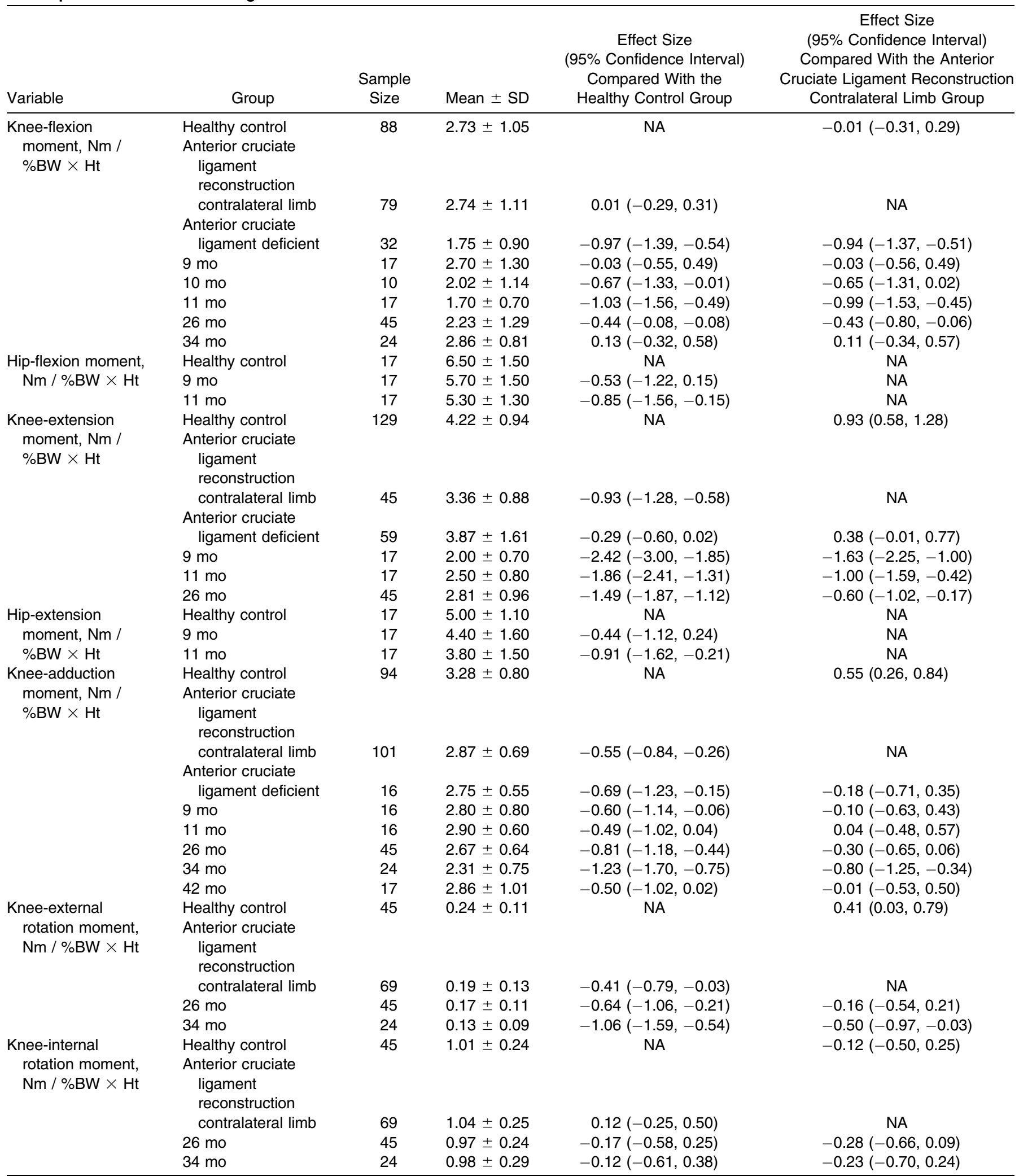

Abbreviation: NA, no data available for group comparison.

Symmetry is an increasingly common indicator of optimal or, in some cases, acceptable muscle function ${ }^{6}$ and movement patterns ${ }^{57,67}$ after ACL injury and ACLR. When the data were collapsed across time since surgery, we noted several cases in which the data for the ACLR limb at a specific time point differed from the composite mean for the contralateral limb collapsed across studies; however, comparisons within individual time points showed that 
Table 3. Peak Sagittal-, Frontal-, and Transverse-Plane Knee and Hip Kinematics After Anterior Cruciate Ligament Injury and Subsequent Anterior Cruciate Ligament Reconstruction Continued on Next Page

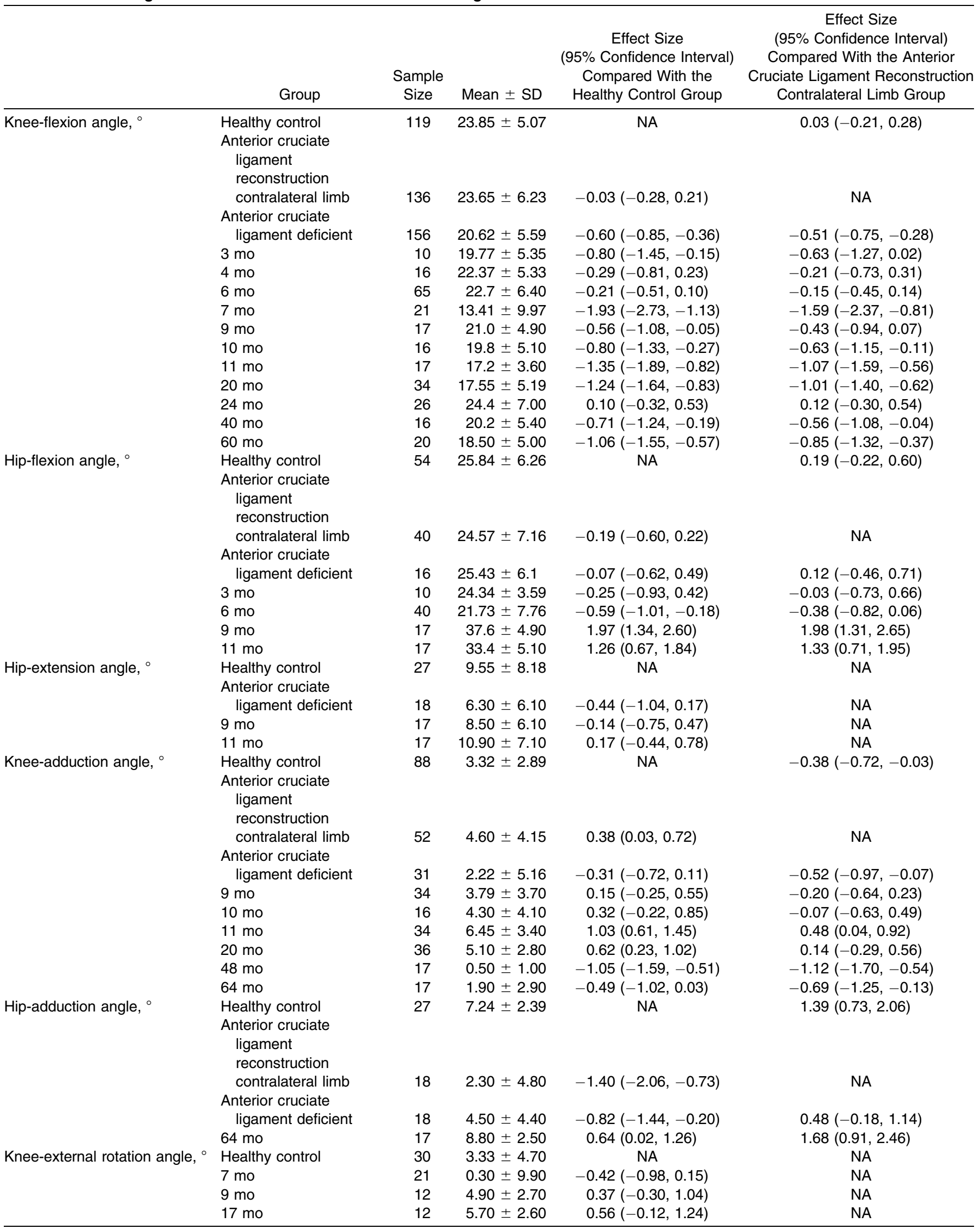




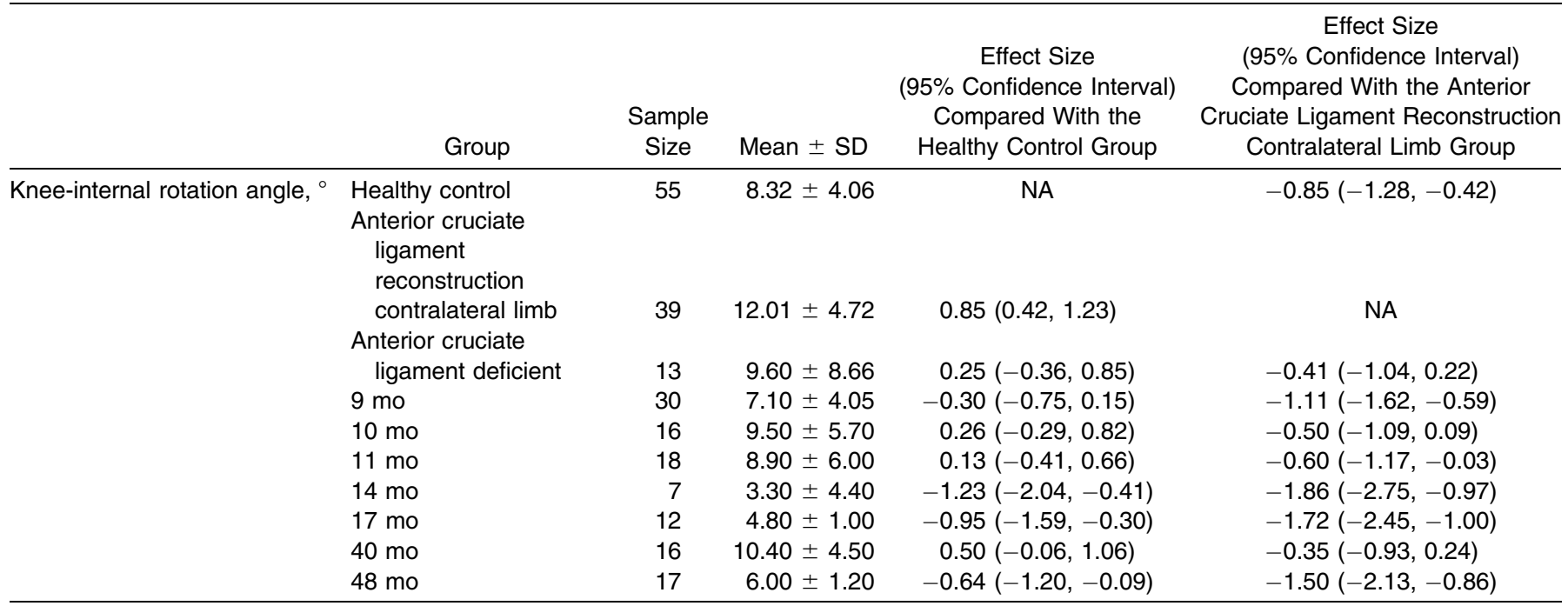

Abbreviation: NA, no data available for group comparison.

between-limbs symmetry was largely maintained (Figures 2 through 5). Except for peak knee-flexion angle at 6 months after ACLR and peak knee-flexion moment at 34 months after ACLR, we found no examples of within-time-point limb asymmetries that were different. This appears to be advantageous, but based on the previously described differences between individuals with ACLR and healthy individuals, this lack of asymmetry may indicate a negative effect of ACL injury on the contralateral limb rather than an advantageous adaptation in the involved limb.
The risk of ACL graft failure and contralateral ACL injury is greatest during the first 24 months after ACLR. ${ }^{12,68}$ The increased injury risk has been linked to several factors, including poor muscle function, lack of neuromuscular control, and alterations in lower extremity movement patterns, among others. ${ }^{12,68}$ Although limited to walking gait, our findings indicate that both frontal- and sagittalplane kinematics and kinetics are altered after initial ACL injury and in the first 36 months after ACLR. Unfortunately, based on a lack of available long-term outcome data, it

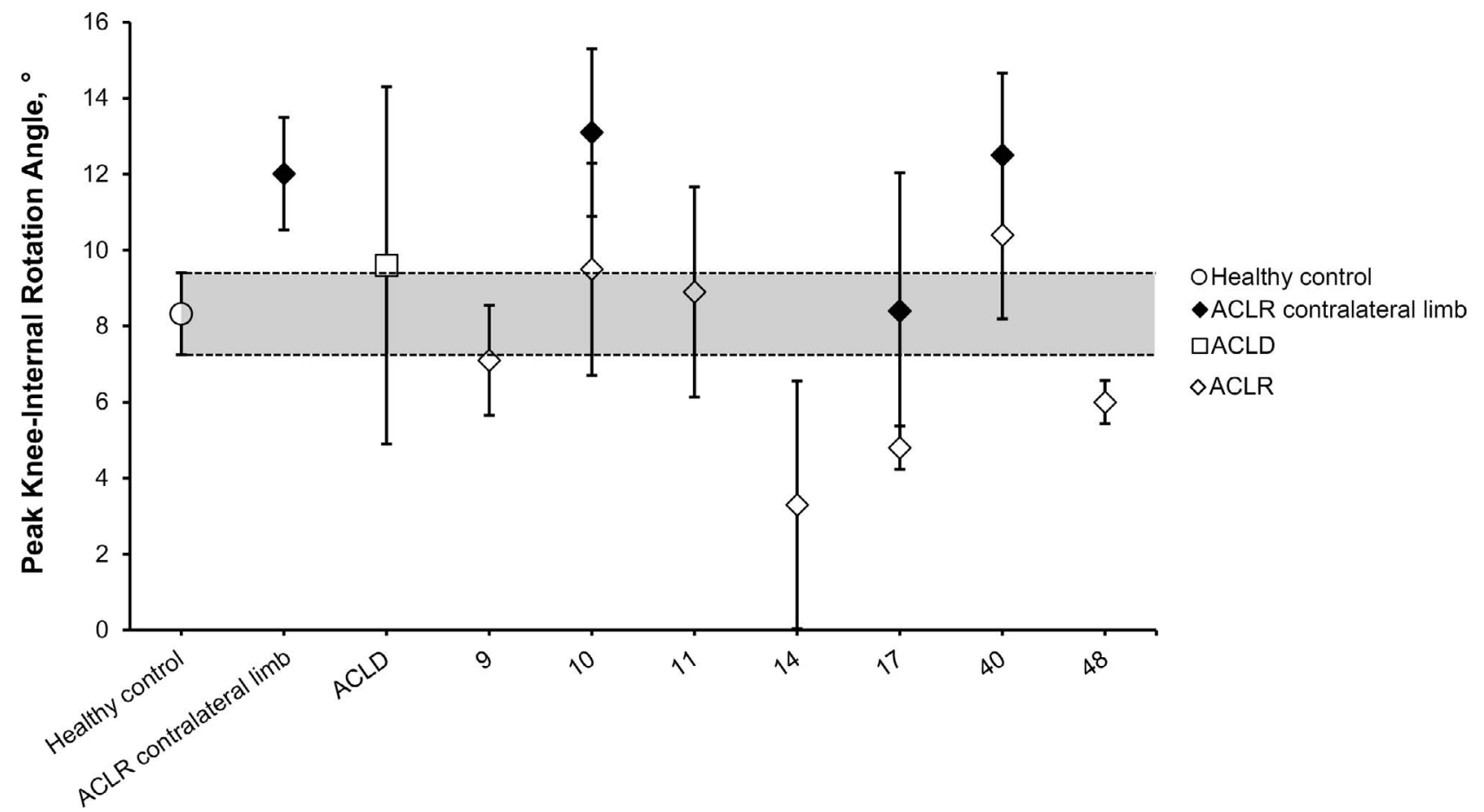

Group

Time After ACLR, mo

Figure 5. Peak knee-internal rotation angle point estimates with $95 \%$ confidence intervals for the healthy control group, anterior cruciate ligament reconstruction (ACLR) contralateral limb group, anterior cruciate ligament deficient (ACLD) group, and each reported time point after ACLR during the stance phase of walking gait. The $95 \%$ confidence interval for the healthy control group is represented using a shaded bar for comparison to all groups and time points. 
remains difficult to determine whether these alterations persist after this initial high-risk period or whether the hypothesized alterations in lower extremity kinematics and kinetics lead to long-term knee-joint cartilage degeneration. Given the currently available evidence, drawing conclusions about gait patterns at long-term follow-up in the population with ACLR remains difficult. ${ }^{14,16,69}$

Several key limitations in the literature we reviewed and the methods we used should be considered. Most importantly, the studies were largely case control or cohort, with very few providing a prospective approach to tracking biomechanical outcomes after ACL injury or ACLR. ${ }^{18,19,32,46}$ Compared with other outcomes of interest, such as patientreported knee function or lower extremity muscle function, for which a broad complement of evidence provides prospective and long-term outcomes for these populations, ${ }^{5,70-72}$ the data available for gait biomechanics remain limited. In addition, whereas the average time since ACLR was presented, several researchers reported substantial variability around the mean estimate of this variable. We presented specific time points throughout the Results section, but not all participants from the included studies were tested at the specific time-points presented. We chose this approach to more clearly aggregate data in the weighted-mean and effect-size calculations while enabling the reader to better understand the progression of biomechanical variables over time. This included single-point estimates for ACLD rather than a timeline for gait mechanics. Time from injury may alter gait mechanics, but our purpose was to compare knee and hip mechanics during walking gait in individuals after ACLR with healthy individuals, the contralateral limb, and individuals with ACLD. In future reviews, investigators should also consider time from injury in both the ACLR and ACLD groups to more comprehensively describe the natural history of lower extremity gait patterns from preinjury through long-term outcomes after ACLR. We did not consider ground reaction forces; however, changes to ground reaction forces would directly affect altered joint kinetics. In future reviews, researchers should consider altered ground reaction forces during walking gait. Substantial variability was present in the methods that each laboratory used to calibrate, collect, and analyze the motion-capture data presented in this review. Furthermore, each laboratory used different walking speeds, with most allowing individuals to self-select their walking speed. This may have substantially affected the quality of the data from each study, but the large number of included studies and relative consistency of findings within each variable of interest should have counteracted this potential confounding factor.

\section{CONCLUSIONS}

Alterations in frontal- and sagittal-plane walking kinematics and kinetics observed early ( $<12$ months) after ACLR persisted into the midterm (12-36 months) followup period. Despite clearance to return to physical activity after ACLR, these gait patterns do not appear to normalize over time, which may indicate that the current approach to rehabilitation and assessment before return to activity is not adequately identifying individuals with dysfunctional movement patterns.

\section{REFERENCES}

1. Mall NA, Chalmers PN, Moric M, et al. Incidence and trends of anterior cruciate ligament reconstruction in the United States. $\mathrm{Am} \mathrm{J}$ Sports Med. 2014;42(10):2363-2370.

2. Ardern CL, Webster KE, Taylor NF, Feller JA. Return to sport following anterior cruciate ligament reconstruction surgery: a systematic review and meta-analysis of the state of play. Br J Sports Med. 2011;45(7):596-606.

3. Oiestad BE, Holm I, Engebretsen L, Risberg MA. The association between radiographic knee osteoarthritis and knee symptoms, function and quality of life 10-15 years after anterior cruciate ligament reconstruction. Br J Sports Med. 2011;45(7):583-588.

4. Ardern CL, Webster KE, Taylor NF, Feller JA. Return to the preinjury level of competitive sport after anterior cruciate ligament reconstruction surgery: two-thirds of patients have not returned by 12 months after surgery. Am J Sports Med. 2011;39(3):538-543.

5. Otzel DM, Chow JW, Tillman MD. Long-term deficits in quadriceps strength and activation following anterior cruciate ligament reconstruction. Phys Ther Sport. 2015;16(1):22-28.

6. Kuenze CM, Hertel J, Weltman A, Diduch D, Saliba SA, Hart JM. Persistent neuromuscular and corticomotor quadriceps asymmetry after anterior cruciate ligament reconstruction. J Athl Train. 2015; 50(3):303-312.

7. Gardinier ES, Manal K, Buchanan TS, Snyder-Mackler L. Gait and neuromuscular asymmetries after acute anterior cruciate ligament rupture. Med Sci Sports Exerc. 2012;44(8):1490-1496.

8. Kuenze C, Hertel J, Hart JM. Effects of exercise on lower extremity muscle function after anterior cruciate ligament reconstruction. $J$ Sport Rehabil. 2013;22(1):33-40.

9. Di Stasi SL, Logerstedt D, Gardinier ES, Snyder-Mackler L. Gait patterns differ between ACL-reconstructed athletes who pass returnto-sport criteria and those who fail. Am J Sports Med. 2013;41(6): 1310-1318.

10. Yamazaki J, Muneta T, Ju YJ, Sekiya I. Differences in kinematics of single leg squatting between anterior cruciate ligament-injured patients and healthy controls. Knee Surg Sports Traumatol Arthrosc. 2010;18(1):56-63.

11. Herrington L, Hatcher J, Hatcher A, McNicholas M. A comparison of Star Excursion Balance Test reach distances between ACL deficient patients and asymptomatic controls. Knee. 2009;16(2):149-152.

12. Paterno MV, Rauh MJ, Schmitt LC, Ford KR, Hewett TE. Incidence of second ACL injuries 2 years after primary ACL reconstruction and return to sport. Am J Sports Med. 2014;42(7):1567-1573.

13. Barenius B, Ponzer S, Shalabi A, Bujak R, Norlen L, Eriksson K. Increased risk of osteoarthritis after anterior cruciate ligament reconstruction: a 14-year follow-up study of a randomized controlled trial. Am J Sports Med. 2014;42(5):1049-1057.

14. Andriacchi TP, Mundermann A. The role of ambulatory mechanics in the initiation and progression of knee osteoarthritis. Curr Opin Rheumatol. 2006;18(5):514-518.

15. Fu RZ, Lin DD. Surgical and biomechanical perspectives on osteoarthritis and the ACL deficient knee: a critical review of the literature. Open Orthop J. 2013;7:292-300.

16. Andriacchi TP, Koo S, Scanlan SF. Gait mechanics influence healthy cartilage morphology and osteoarthritis of the knee. J Bone Joint Surg Am. 2009;91(suppl 1):95-101.

17. Webster KE, McClelland JA, Palazzolo SE, Santamaria LJ, Feller JA. Gender differences in the knee adduction moment after anterior cruciate ligament reconstruction surgery. Br J Sports Med. 2012; 46(5):355-359.

18. Timoney JM, Inman WS, Quesada PM, et al. Return of normal gait patterns after anterior cruciate ligament reconstruction. Am J Sports Med. 1993;21(6):887-889.

19. Shin CS, Chaudhari AM, Dyrby CO, Andriacchi TP. Influence of patellar ligament insertion angle on quadriceps usage during walking 
in anterior cruciate ligament reconstructed subjects. J Orthop Res. 2009;27(6):730-735.

20. Andriacchi TP. Valgus alignment and lateral compartment knee osteoarthritis: a biomechanical paradox or new insight into knee osteoarthritis? Arthritis Rheum. 2013;65(2):310-313.

21. Hurwitz DE, Ryals AR, Block JA, Sharma L, Schnitzer TJ, Andriacchi TP. Knee pain and joint loading in subjects with osteoarthritis of the knee. J Orthop Res. 2000;18(4):572-579.

22. Sharma L, Hurwitz DE, Thonar EJ, et al. Knee adduction moment, serum hyaluronan level, and disease severity in medial tibiofemoral osteoarthritis. Arthritis Rheum. 1998;41(7):1233-1240.

23. Downs SH, Black N. The feasibility of creating a checklist for the assessment of the methodological quality both of randomised and non-randomised studies of health care interventions. $J$ Epidemiol Community Health. 1998;52(6):377-384.

24. de Morton NA. The PEDro scale is a valid measure of the methodological quality of clinical trials: a demographic study. Aust $J$ Physiother. 2009;55(2):129-133.

25. Lohr KN. Rating the strength of scientific evidence: relevance for quality improvement programs. Int J Qual Health Care. 2004;16(1): 9-18.

26. Chen $\mathrm{CH}$, Li JS, Hosseini A, Gadikota HR, Gill TJ, Li G. Anteroposterior stability of the knee during the stance phase of gait after anterior cruciate ligament deficiency. Gait Posture. 2012;35(3): 467-471.

27. Butler RJ, Minick KI, Ferber R, Underwood F. Gait mechanics after ACL reconstruction: implications for the early onset of knee osteoarthritis. Br J Sports Med. 2009;43(5):366-370.

28. Di Stasi SL, Snyder-Mackler L. The effects of neuromuscular training on the gait patterns of ACL-deficient men and women. Clin Biomech (Bristol, Avon). 2012;27(4):360-365.

29. Ferber R, Osternig LR, Woollacott MH, Wasielewski NJ, Lee JH. Gait mechanics in chronic ACL deficiency and subsequent repair. Clin Biomech (Bristol, Avon). 2002;17(4):274-285.

30. Georgoulis AD, Papadonikolakis A, Papageorgiou CD, Mitsou A, Stergiou N. Three-dimensional tibiofemoral kinematics of the anterior cruciate ligament-deficient and reconstructed knee during walking. Am J Sports Med. 2003;31(1):75-79.

31. Houck JR, De Haven KE, Maloney M. Influence of anticipation on movement patterns in subjects with ACL deficiency classified as noncopers. J Orthop Sports Phys Ther. 2007;37(2):56-64.

32. Knoll Z, Kocsis L, Kiss RM. Gait patterns before and after anterior cruciate ligament reconstruction. Knee Surg Sports Traumatol Arthrosc. 2004;12(1):7-14.

33. Lewek M, Rudolph K, Axe M, Snyder-Mackler L. The effect of insufficient quadriceps strength on gait after anterior cruciate ligament reconstruction. Clin Biomech (Bristol, Avon). 2002;17(1):56-63.

34. Lindstrom M, Fellander-Tsai L, Wredmark T, Henriksson M. Adaptations of gait and muscle activation in chronic ACL deficiency. Knee Surg Sports Traumatol Arthrosc. 2010;18(1):106-114.

35. Noyes FR, Schipplein OD, Andriacchi TP, Saddemi SR, Weise M. The anterior cruciate ligament-deficient knee with varus alignment: an analysis of gait adaptations and dynamic joint loadings. $\mathrm{Am} \mathrm{J}$ Sports Med. 1992;20(6):707-716.

36. Patterson MR, Delahunt E, Caulfield B. Peak knee adduction moment during gait in anterior cruciate ligament reconstructed females. Clin Biomech (Bristol, Avon). 2014;29(2):138-142.

37. Roberts CS, Rash GS, Honaker JT, Wachowiak MP, Shaw JC. A deficient anterior cruciate ligament does not lead to quadriceps avoidance gait. Gait Posture. 1999;10(3):189-199.

38. Roewer BD, Di Stasi SL, Snyder-Mackler L. Quadriceps strength and weight acceptance strategies continue to improve two years after anterior cruciate ligament reconstruction. J Biomech. 2011;44(10): 1948-1953.

39. Torry MR, Decker MJ, Ellis HB, Shelburne KB, Sterett WI, Steadman JR. Mechanisms of compensating for anterior cruciate ligament deficiency during gait. Med Sci Sports Exerc. 2004;36(8): 1403-1412.

40. Webster KE, Wittwer JE, O'Brien J, Feller JA. Gait patterns after anterior cruciate ligament reconstruction are related to graft type. Am J Sports Med. 2005;33(2):247-254.

41. Webster KE, Feller JA. The knee adduction moment in hamstring and patellar tendon anterior cruciate ligament reconstructed knees. Knee Surg Sports Traumatol Arthrosc. 2012;20(11):2214-2219.

42. Webster KE, Feller JA. Alterations in joint kinematics during walking following hamstring and patellar tendon anterior cruciate ligament reconstruction surgery. Clin Biomech (Bristol, Avon). 2011; 26(2):175-180.

43. Zabala ME, Favre J, Scanlan SF, Donahue J, Andriacchi TP. Threedimensional knee moments of ACL reconstructed and control subjects during gait, stair ascent, and stair descent. $J$ Biomech. 2013;46(3):515-520.

44. Sato K, Maeda A, Takano Y, Matsuse H, Ida H, Shiba N. Relationship between static anterior laxity using the KT-1000 and dynamic tibial rotation during motion in patients with anatomical anterior cruciate ligament reconstruction. Kurume Med J. 2013; 60(1): $1-6$.

45. Wang H, Fleischli JE, Zheng NN. Transtibial versus anteromedial portal technique in single-bundle anterior cruciate ligament reconstruction: outcomes of knee joint kinematics during walking. Am J Sports Med. 2013;41(8):1847-1856.

46. Knoll Z, Kiss RM, Kocsis L. Gait adaptation in ACL deficient patients before and after anterior cruciate ligament reconstruction surgery. J Electromyogr Kinesiol. 2004;14(3):287-294.

47. Noehren B, Wilson H, Miller C, Lattermann C. Long-term gait deviations in anterior cruciate ligament-reconstructed females. Med Sci Sports Exerc. 2013;45(7):1340-1347.

48. Webster KE, Feller JA, Wittwer JE. Longitudinal changes in knee joint biomechanics during level walking following anterior cruciate ligament reconstruction surgery. Gait Posture. 2012;36(2):167-171.

49. Lee SK. The effect of a vertical load on gluteus medius activity and gait characteristics during walking. J Phys Ther Sci. 2013;25(11): 1397-1399.

50. Lee SK, Jung JM, Lee SY. Gluteus medius muscle activation on stance phase according to various vertical load. J Back Musculoskelet Rehabil. 2013;26(2):159-161.

51. Thomas AC, Villwock M, Wojtys EM, Palmieri-Smith RM. Lower extremity muscle strength after anterior cruciate ligament injury and reconstruction. J Athl Train. 2013;48(5):610-620.

52. Dalton EC, Pfile KR, Weniger GR, Ingersoll CD, Herman D, Hart JM. Neuromuscular changes after aerobic exercise in people with anterior cruciate ligament reconstructed knees. J Athl Train. 2011; 46(5):476-483.

53. Lepley LK. Deficits in quadriceps strength and patient-oriented outcomes at return to activity after ACL reconstruction: a review of the current literature. Sports Health. 2015;7(3):231-238.

54. Ardern CL, Taylor NF, Feller JA, Webster KE. Fifty-five per cent return to competitive sport following anterior cruciate ligament reconstruction surgery: an updated systematic review and metaanalysis including aspects of physical functioning and contextual factors. Br J Sports Med. 2014;48(21):1543-1552.

55. Brophy RH, Schmitz L, Wright RW, et al. Return to play and future ACL injury risk after ACL reconstruction in soccer athletes from the Multicenter Orthopaedic Outcomes Network (MOON) group. Am J Sports Med. 2012;40(11):2517-2522.

56. Goetschius J, Kuenze CM, Saliba S, Hart JM. Reposition acuity and postural control after exercise in anterior cruciate ligament reconstructed knees. Med Sci Sports Exerc. 2013;45(12):2314 2321.

57. Augustsson J, Thomee R, Karlsson J. Ability of a new hop test to determine functional deficits after anterior cruciate ligament 
reconstruction. Knee Surg Sports Traumatol Arthrosc. 2004;12(5): 350-356.

58. Castanharo R, da Luz BS, Bitar AC, D’Elia CO, Castropil W, Duarte M. Males still have limb asymmetries in multijoint movement tasks more than 2 years following anterior cruciate ligament reconstruction. J Orthop Sci. 2011;16(5):531-535.

59. Hart JM, Ko JW, Konold T, Pietrosimone B. Sagittal plane knee joint moments following anterior cruciate ligament injury and reconstruction: a systematic review. Clin Biomech (Bristol, Avon). 2010;25(4): 277-283.

60. Alnahdi AH, Zeni JA, Snyder-Mackler L. Gait after unilateral total knee arthroplasty: frontal plane analysis. J Orthop Res. 2011;29(5): $647-652$.

61. Foroughi N, Smith R, Vanwanseele B. The association of external knee adduction moment with biomechanical variables in osteoarthritis: a systematic review. Knee. 2009;16(5):303-309.

62. Oiestad BE, Engebretsen L, Storheine K, Risberg MA. Knee osteoarthritis after anterior cruciate injury: a systematic review. $\mathrm{Am}$ J Sports Med. 2009;37(7):1434-1443.

63. Bassett DR Jr, Wyatt HR, Thompson H, Peters JC, Hill JO. Pedometer-measured physical activity and health behaviors in U.S. adults. Med Sci Sports Exerc. 2010;42(10):1819-1825.

64. Zwolski C, Schmitt LC, Quatman-Yates C, Thomas S, Hewett TE, Paterno MV. The influence of quadriceps strength asymmetry on patient-reported function at time of return to sport after anterior cruciate ligament reconstruction. Am J Sports Med. 2015;43(9): 2242-2249.

65. Mills K, Hunt MA, Ferber R. Biomechanical deviations during level walking associated with knee osteoarthritis: a systematic review and meta-analysis. Arthritis Care Res (Hoboken). 2013;65(10):16431665.

66. Chang A, Hayes K, Dunlop D, et al. Thrust during ambulation and the progression of knee osteoarthritis. Arthritis Rheum. 2004;50(12): 3897-3903.

67. Ericsson YB, Roos EM, Frobell RB. Lower extremity performance following ACL rehabilitation in the KANON-trial: impact of reconstruction and predictive value at 2 and 5 years. Br J Sports Med. 2013;47(15):980-985.

68. Paterno MV, Rauh MJ, Schmitt LC, Ford KR, Hewett TE. Incidence of contralateral and ipsilateral anterior cruciate ligament (ACL) injury after primary ACL reconstruction and return to sport. Clin J Sport Med. 2012;22(2):116-121.

69. Andriacchi TP, Briant PL, Bevill SL, Koo S. Rotational changes at the knee after ACL injury cause cartilage thinning. Clin Orthop Relat Res. 2006;442:39-44.

70. Dunn WR, Spindler KP, MOON Consortium. Predictors of activity level 2 years after anterior cruciate ligament reconstruction (ACLR): a Multicenter Orthopaedic Outcomes Network (MOON) ACLR cohort study. Am J Sports Med. 2010;38(10):2040-2050.

71. Spindler KP, Huston LJ, Wright RW, et al. The prognosis and predictors of sports function and activity at minimum 6 years after anterior cruciate ligament reconstruction: a population cohort study. Am J Sports Med. 2011;39(2):348-359.

72. Wright RW, Dunn WR, Amendola A, et al. Risk of tearing the intact anterior cruciate ligament in the contralateral knee and rupturing the anterior cruciate ligament graft during the first 2 years after anterior cruciate ligament reconstruction: a prospective MOON cohort study. Am J Sports Med. 2007;35(7):11311134.

Address correspondence to Lindsay V. Slater, PhD, Department of Kinesiology, The University of Virginia, 210 South Emmet Street, Suite 203, PO Box 400407, Charlottesville, VA 22904-4407. Address e-mail to ls4zj@virginia.edu. 